- Case Report

\title{
Escitalopram-Induced Amenorrhea and False Positive Urine Pregnancy Test
}

\author{
Vithyalakshmi Selvaraj ${ }^{1,2, *}$, Siv Hour ${ }^{1}$, Palanikumar Gunasekar ${ }^{3}$, Caron Gray², James F. Smith ${ }^{2}$ \\ 'Department of Psychiatry, Creighton University School of Medicine, Omaha, USA \\ ${ }^{2}$ Department of Obstetrics and Gynecology, Creighton University School of Medicine, Omaha, USA \\ ${ }^{3}$ Department of Biomedical Sciences, Creighton University School of Medicine, Omaha, USA
}

Escitalopram is a selective serotonin reuptake inhibitor antidepressant approved by the Food and Drug Administration for the treatment of major depressive disorder and generalized anxiety disorder. A 34-year-old female patient with major depressive disorder developed amenorrhea and had a false-positive urine pregnancy test after initiation of escitalopram treatment. To our knowledge, no published case report of amenorrhea and false-positive urine pregnancy tests in women taking escitalopram exists. This case report suggests that women of child-bearing age should be carefully monitored for amenorrhea while they are on an antidepressant treatment regimen.

Keywords: Selective Serotonin Reuptake Inhibitors; Hyperprolactinemia; Amenorrhea; Immunologic Pregnancy Tests; Case Reports 


\section{INTRODUCTION}

There have been a few case reports on selective serotonin reuptake inhibitor (SSRI)-induced hyperprolactinemia, ${ }^{1-3)}$ which may lead to secondary amenorrhea; however, to our knowledge, this is the first case in which a false-positive urine pregnancy test was observed following initiation of escitalopram treatment. This case report suggests that women of child-bearing age taking antidepressants should be closely monitored.

\section{CASE REPORT}

Miss L is a 34-year-old Caucasian female with a history of premenstrual dysphoric disorder and panic disorder. She was started on citalopram in 2011, which helped her manage her depression. She then returned to the clinic in 2013 for worsening signs of depression and anxiety following the murder of her brother. She was started on alprazolam $0.5 \mathrm{mg}$ four times a day when necessary (prn) and zolpidem $5 \mathrm{mg} \mathrm{ev}$ ery hour of sleep (QHS) prn for anxiety and sleep disorders, respectively. Maximum citalopram dosage was reached at $40 \mathrm{mg}$ in the morning; hence, mirtazapine was added to the treatment regimen for worsening affective symptoms. Mirtazapine was titrated to $30 \mathrm{mg}$ QHS, after which her mood improved significantly. Citalopram treatment was discontinued because mirtazapine was effective, and her wellbeing was maintained for over 12 months on mirtazapine monotherapy. In 2014, she developed panic attacks again, following situational stressors at work and home. Subsequently, she was started on escitalopram $10 \mathrm{mg}$ daily (QD) as an augmenting agent for her worsening depressive symptoms; after 3 months, the escitalopram dosage was increased to $20 \mathrm{mg}$ QD. She presented for follow-up at 1 month after starting on $20 \mathrm{mg}$ QD escitalopram and reported that she had missed her period after initiating escitalopram and had subsequently completed a home pregnancy test, which was positive. Upon evaluation by her primary care physician, it was determined that her body mass index had not changed and there was no change in her mood. Results of a serum pregnancy test were negative. She was advised by her physician to discontinue escitalopram because it was the only new medication that she had recently started. Two weeks after discontinuing escitalopram, her menses resumed.

\section{DISCUSSION}

There have been a few reported cases of SSRI-induced hyperprolactinemia and development of galactorrhea while on escitalopram. ${ }^{1-3)}$ Serotonin may stimulate prolactin release either directly through postsynaptic 5-hydroxytrypamine (5-HT) receptors in the hypothalamus or indirectly via 5-HT mediated-inhibition of tuberoinfundibular dopaminergic neurons. ${ }^{4)}$ One study comparing citalopram and escitalopram showed that after a single dose, escitalopram acted centrally (and not peripherally) and increased prolactin and cortisol levels while also exhibiting successful blockade of cortisol following admin- istration of dexamethasone. ${ }^{5)}$

Miss L, who reports a history of regular menses, is thought to have developed amenorrhea as a result of escitalopram treatment. The mechanism behind the development of amenorrhea may be a result of serotonin's effect of increasing prolactin levels. Unfortunately, the patient's prolactin levels were not ascertained at her follow-up appointment with her mental health provider (after having initiated escitalopram) because two months had already passed since discontinuation of escitalopram. Elevated prolactin levels could cause amenorrhea by suppressing hypothalamic gonadotropin-releasing hormone, thereby decreasing production of gonadotropins such as luteinizing hormone (LH) and follicle-stimulating hormone.

LH may play a role in false-positive urine pregnancy tests in patients receiving chlorpromazine. ${ }^{6)}$ One study showed secretion of the posterior pituitary (neurohypophysial) hormones vasopressin and oxytocin through stimulation of serotonergic receptors in rats; in particular, the 5-HT1A receptor was involved in serotonergic stimulation of oxytocin secretion. ${ }^{7)}$ Most studies investigating the role of oxytocin in LH regulation have been conducted in rats and show that oxytocin elicits concentration-dependent secretion of $\mathrm{LH}^{8}{ }^{8)} \mathrm{In}$ a small study, an oxytocin antagonist affected the ovulatory cycle of non-pregnant women, pointing to a role for oxytocin in the physiological processes of LH regulation in women..$^{9)}$ In the case of Miss L, the serotonergic effects of escitalopram (which may be unique in its central-acting nature) may have been involved in an increase in oxytocin and subsequently an increase in LH. LH apparently cross-reacts with the antichorionic gonadotropin antibody used in immunologic urine pregnancy tests and can cause a false-positive result. ${ }^{6)}$ Potential causes of false-positive urine pregnancy tests include medical conditions such as malignancy and proteinuria. This case report suggests that women of child-bearing age should be carefully monitored while they are on an antidepressants.

\section{CONFLICT OF INTEREST}

No potential conflict of interest relevant to this article was reported.

\section{REFERENCES}

1. Gulsun M, Evrensel A, Verim S. Galactorrhea during escitalopram treatment: a case report. Bull Clin Psychopharmacol 2006;16:39-41.

2. Gulsun M, Algul A, Semiz UB, Ates MA, Doruk A, Ebrinc S, et al. A case with euprolactinemic galactorrhea induced by escitalopram. Int J Psychiatry Med 2007;37:275-8.

3. Mondal S, Saha I, Das S, Ganguly A, Das D, Tripathi SK. A new logical insight and putative mechanism behind fluoxetine-induced amenorrhea, hyperprolactinemia and galactorrhea in a case series. Ther Adv Psychopharmacol 2013;3:322-34.

4. Nicholas L, Dawkins K, Golden RN. Psychoneuroendocrinology of depression: prolactin. Psychiatr Clin North Am 1998;21:341-58.

5. Hawken ER, Owen JA, Hudson RW, Delva NJ. Specific effects of escitalopram on neuroendocrine response. Psychopharmacology (Berl) 
2009;207:27-34.

6. Ravel R, Riekers HG, Goldstein BJ. Effects of certain psychotropic drugs on immunologic pregnancy tests. Am J Obstet Gynecol 1969;105:1222-5.

7. Jorgensen H, Riis M, Knigge U, Kjaer A, Warberg J. Serotonin receptors involved in vasopressin and oxytocin secretion. J Neuroendocrinol 2003;15:242-9.
8. Uvnas-Moberg K, Bjokstrand E, Hillegaart V, Ahlenius S. Oxytocin as a possible mediator of SSRI-induced antidepressant effects. Psychopharmacology (Berl) 1999;142:95-101.

9. Evans JJ, Reid RA, Wakeman SA, Croft LB, Benny PS. Evidence that oxytocin is a physiological component of LH regulation in non-pregnant women. Hum Reprod 2003;18:1428-31. 